\title{
A Case of Secondary Rectal Intramural Hematoma Triggered by Aspiration for Endoscopic Mucosal Resection
}

\section{ISSN: 2637-7632}

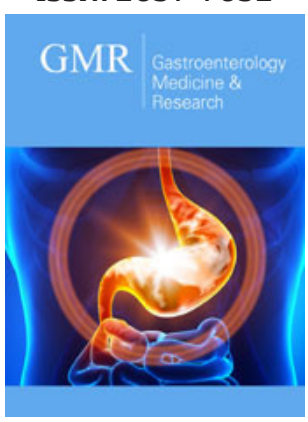

*Corresponding author: Masashi Okuro, Department of Geriatric Medicine, Kanazawa Medical University, Uchinada, Kahoku-gun, Ishikawa 9200293, Japan

Submission: 㖰 March 24, 2021

Published: 眥April 07, 2021

Volume 5 - Issue 5

How to cite this article: Kazu Hamada, Tohru Itoh, Ken Kawaura, Hiroaki Kuno, Junji Kamai, Rika Kobayasi, et al. A Case of Secondary Rectal Intramural Hematoma Triggered by Aspiration for Endoscopic Mucosal Resection. Gastro Med Res. 5(5). GMR. 000623. 2021.

DOI: 10.31031/GMR.2021.05.000623

Copyright@ Masashi Okuro, This article is distributed under the terms of the Creative Commons Attribution 4.0 International License, which permits unrestricted use and redistribution provided that the original author and source are credited.
Kazu Hamada ${ }^{1,2}$, Tohru Itoh ${ }^{2}$, Ken Kawaura ${ }^{2}$, Hiroaki Kuno ${ }^{2}$, Junji Kamai ${ }^{2}$, Rika Kobayasi $^{2}$, Sadahumi Azukisawa ${ }^{2}$, Hidekazu Kitakata ${ }^{2}$, Yuta Igarashi ${ }^{1}$, Kumie Kodera $^{1}$, Tazuo Okuno ${ }^{1}$, Takuro Morita ${ }^{1}$, Hiroshi Yano ${ }^{1}$, Toshihiro Higashikawa ${ }^{1}$, Osamu Iritani ${ }^{1}$, Kunimitsu Iwai ${ }^{1}$, Shigeto Morimoto ${ }^{1}$ and Masashi Okuro ${ }^{1 *}$

${ }^{1}$ Department of Geriatric Medicine, Kanazawa Medical University, Japan

${ }^{2}$ Department of Gastroenterological Endoscopy, Kanazawa Medical University, Japan

\begin{abstract}
Total colonoscopy was performed on an 89-year-old man who was not treated with anticoagulation therapy. He had developed purpura in the rectum below the peritoneal reflection by the aspiration for endoscopic mucosal resection to rectal polyp. The purpura resulted in spontaneous cessation, but he had lower abdominal pain thereafter. He was diagnosed to have intramural hematoma of the rectum from rectosigmoid to above the peritoneal reflection by computed tomography and sigmoidoscopy. It disappeared with conservative therapy. We infer that it was secondary rectal intramural hematoma triggered by excessive straining against purpura.
\end{abstract}

Keywords: Rectum; Intramural hematoma; Endoscopic mucosal resection; Abdominal pain; Straining

Abbreviations: IH: Intramural Hematoma; TCS: Total Colonoscopy; RB: Rectum below the peritoneal reflection EMR: Endoscopic Mucosal Resection, PT-INR: Prothrombin Time-International Normalized Ratio; APTT: Activated Partial Thromboplastin Time; CT: Computed Tomography; RS: Rectum from Rectosigmoid RA: Rectum above the Peritoneal Reflection

\section{Introduction}

Intramural Hematoma ( $\mathrm{IH})$ of intestine is a rare condition [1] that may develop from esophagus to rectum. It often occurs in patients with anticoagulation therapy [2-4], hemophilia [5], amyloidosis [6], and Ehlers-Danlos syndrome [7], and occurs rarely without coagulation disorder. We report here a case of secondary rectal IH triggered by aspiration for endoscopic mucosal resection without coagulation disorder.

\section{Case Presentation}

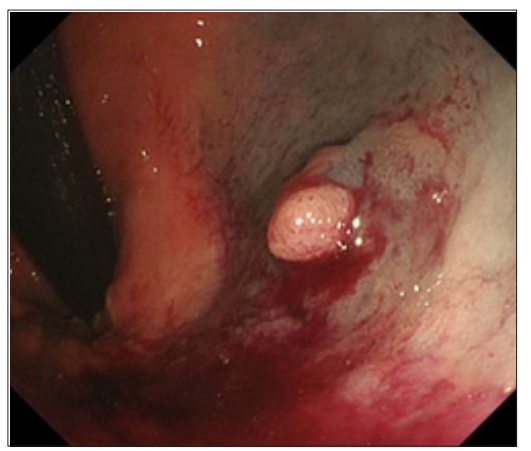

Figure 1: Total colonoscopy showed a polyp which was $5 \mathrm{~mm}$ in size on the rectum below the peritoneal reflection; $39 \mathrm{~cm}$ from the anus. Purpura was caused by the aspiration for endoscopic mucosal resection.

He was treated with medicines for high blood pressure hypertension, hyperuricemia, iron deficiency anemia, and insomnia. A cecal cancer was diagnosed in him by Total Colonoscopy (TCS) which was performed to check cause of fecal occult blood. We assessed that operation was necessary to cure the lesion. In addition, there was a semi-pedunculated polyp which 
was $5 \mathrm{~mm}$ size on the rectum below the peritoneal Reflection (Rb) and we tried to perform Endoscopic Mucosal Resection (EMR) for it immediately. However, we interrupted to perform EMR for it because severe purpura was caused by the aspiration for injection to submucosa (Figure 1). We confirmed spontaneous cessation of hemorrhage, and he was admitted to our hospital for observation of the progress on this day. He recognized only slight tenesmus and unpleasant feeling of anus, and his general condition was stable. So that, he was discharged 2 days later.

He repeated straining excessively against these symptoms after TCS. The lower abdominal pain developed from the next day after discharge, and It rapidly got worse. Therefore, he visited our hospital again 4 days after TCS. On physical examination, blood pressure was $134 / 72 \mathrm{mmHg}$, pulse was 66 beats per minute/regular, and temperature was 37.1 degrees. The auscultatory findings of lungs and heart were normal. The abdomen was soft-flat, bowel sound was normal, and there was no tenderness. In laboratory data, hemoglobin level was $12.0 \mathrm{~g} / \mathrm{dL}$ which was $12.3 \mathrm{~g} / \mathrm{dL}$ before TCS, and there was no appearance of anemia was. The platelet count was $19.2 \times 104 / \mathrm{uL}$, prothrombin time-international normalized ratio (PT-INR) was 1.02 and Activated Partial Thromboplastin Time (APTT) was 26.9 seconds, all of which were normal.

The abdominal plain Computed Tomography (CT) showed a large highly absorbed mass in the rectum from Rectosigmoid (RS) to above the peritoneal Reflection (Ra) (Figure 2a). There were no definitive findings to suggest active bleeding with contrastenhanced CT (Figure 2b). Sigmoidoscopy demonstrated a large dark-red mass in the rectum from RS to Ra (Figure 3a). A rectal moderate stenosis was caused by the mass, endoscope passed there easily. On the anal side, there was a polyp which was interrupted to perform EMR the previous time. Purpura in the polyp and the mucosa around it was improved (Figure $3 \mathrm{~b}$ ). We diagnosed rectal IH. Since his general condition was stable and IH was examined as spontaneous cessation with CT, we decided to treat conservatively rather than surgically. He was admitted and received conservative therapy which were bowel rest and intravenous hydration. His symptoms improved in several days. We confirmed that IH did not grow with abdominal CT performed 7 days after admission. He was discharged 13 days later.

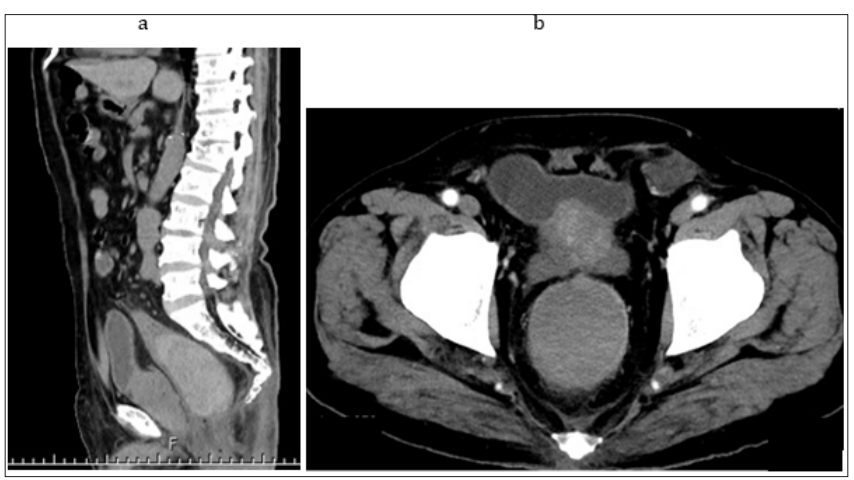

Figure 2:

(a): Sagittal section of abdominal plain computed tomography showed a large highly absorbed mass (white arrows) which was $9 * 9 * 5 \mathrm{~cm}$ in size in the rectum from rectosigmoid to above the peritoneal reflection.

(b): Axial section of abdominal contrast-enhanced computed tomography showed no definitive findings in it (white arrow heads).

a

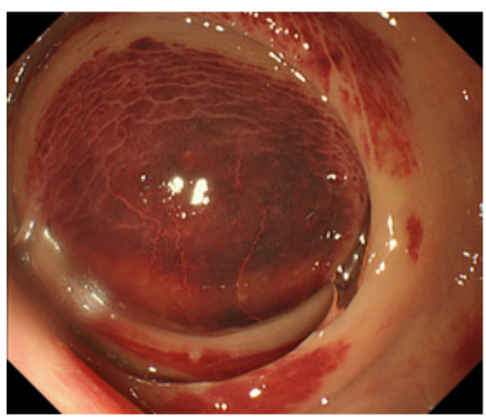

b

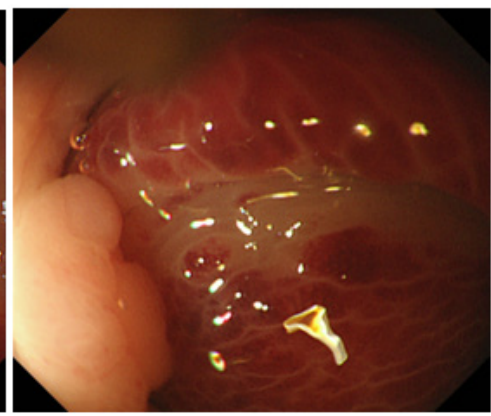

Figure 3:

(a): Sigmoidoscopy showed a large dark-red mass in the rectum above the peritoneal reflection.

(b): On the anal side, sigmoidoscopy showed a polyp (white arrows) which was interrupted to perform endoscopic mucosal resection in the previous time. Purpura in the polyp and the mucosa around it was improved. 
About two months later, sigmoidoscopy demonstrated that rectal IH disappeared (Figure 4). Cecal cancer operation was performed and examined as stage I/radical excision by histological diagnosis. Now, four years later, rectal IH and cecal cancer have not relapsed.

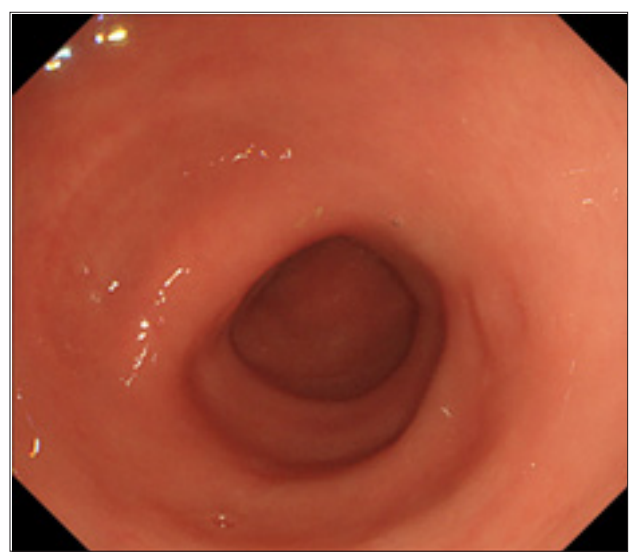

Figure 4: Sigmoidoscopy demonstrated that the rectal mass disappeared about two months later.

\section{Discussion}

IH of intestine often occurs in small intestine and duodenum, whereas colonic IH is rare [8,9]. Its typical symptoms are abdominal pain and nausea/vomiting [1,9]. It is diagnosed by endoscopy, abdominal ultrasonography, and CT $[1,3,10]$. In addition, there is a report that endoscopic ultrasonography was useful for diagnosis of colonic IH [11]. However, it is often difficult to distinguish IH from neoplasm [12]. In this case, we confirmed that there was no rectal IH by TCS several days earlier. It was relatively easy to diagnose rectal mass with IH from his history and sigmoidoscopy/CT findings. The standard treatment for $\mathrm{IH}$ of intestine is conservative therapy if general condition is stable $[3,4,10,13]$, but we should consider operation when it is accompanied by intestinal obstruction and peritonitis $[1,2]$.

The major cause of $\mathrm{IH}$ is abdominal blunt trauma $[3,8,10]$. In addition, it is often caused by coagulation disorder [1-5]. However, this case did not have these factors. There is a report of a colonic IH associated with colon cancer [14], and our case had a cecal cancer. However, we think that it was unrelated among both for our case because there was distance from each other, and it was stage I/radical excision lesion. There were some cases of colonic IH which were caused by endoscopic procedures such as polypectomy $[11,15]$, and tattooing [16]. Because severe purpura was caused by aspiration for EMR several days before rectal IH occurred, we thought that there was a relationship between both in this case. However, purpura by aspiration was located in $\mathrm{Rb}$ whereas IH was located in RS-Ra. In addition, purpura improved when IH appeared. Therefore, it was thought that aspiration did not influence occurrence of IH directly. A non-traumatic rectal IH caused by straining was reported [2], and we guessed that the mechanism in our case was also the same. In summary, purpura by aspiration for EMR gave him some symptoms such as tenesmus and unpleasant feeling of anus, and he repeated straining excessively against them. The straining increased abdominal pressure and rectal muscular contraction. They gave rectum severe stress, and it led to secondary rectal $\mathrm{IH}$. We infer that a case like this is extremely rare, but it is important to recognize that excessive straining may cause rectal IH. For preventing rectal $\mathrm{IH}$, we should perform instruction and prescribe medicine to avoid excessive straining for patients with some conditions, such as colitis, constipation, and rectal purpura caused by aspiration.

\section{Acknowledgement}

Kazu Hamada wrote the manuscript; Masashi Okuro and Ken Kawaura collected data for review; all authors read and approved the final version of the manuscript. We thank all the investigators, staff, and participants associated with this report for helpful discussions during manuscript development.

\section{Conflict of Interest}

The authors declare no conflict of interest.

\section{References}

1. Polat C, Dervisoglu A, Guven H, Kaya E, Malazgirt Z, et al. (2003) Anticoagulant-induced intramural intestinal hematoma. Am J Emerg Med 21(3): 208-211.

2. Li ZL, Wang ZJ, Han JG (2012) Spontaneous perforation of an intramural rectal hematoma: Report of a case. World J Gastroenterol 18(19): 24382440 .

3. Kwon K, Cheung DY, Seo Y, Kim SB, Bae KN, et al. (2014) Supportive management resolved a colonic intramural hematoma in an anticoagulant user. Intern Med 53(14): 1505-1509.

4. Lobo L, Koudki R, Prasad Hl K, Shetty B (2013) Colon obstruction due to an anticoagulant induced intramural haematoma: A Rare Case Report. J Clin Diagn Res 7(4): 739-741.

5. Mittal R, Spero JA, Lewis JH, Taylor F, Ragni MV, et al. (1985) Patterns of gastrointestinal hemorrhage in hemophilia. Gastroenterology 88(2): 515-522.

6. Iijima-Dohi N, Shinji A, Shimizu T, Ishikawa SZ, Mukawa K, et al. (2004) Recurrent gastric hemorrhaging with large submucosal hematomas in a patient with primary AL systemic amyloidosis: Endoscopic and histopathological findings. Intern Med 43(6): 468-472.

7. Kobayashi Y, Kuwabara A, Okamoto H, Sugimura K, Hatakeyama K (2006) Gastric submucosal hematoma and mucosal rupture with Ehlers-Danlos syndrome: a case report. Acta Medica et Biologica 54(2): 59-62.

8. Hughes CE, Conn J Jr, Sherman JO (1977) Intramural hematoma of the gastrointestinal tract. Am J Surg 133(3): 276-279.

9. Tseng CY, Fan JS, Yang SC, Huang HH, Chen JD, et al. (2010) Anticoagulantinduced intramural intestinal hemorrhage. Am J Emerg Med 28(8): 937940 .

10. McClenathan JH, Dabadghav N (2004) Blunt rectal trauma causing intramural rectal hematoma: Report of a case. Dis Colon Rectum 47(3): 380-382.

11. Soejima A, Akahoshi K, Furuno T, Kojima H, Fujimaru T, et al. (2001) A case of polypectomy induced colonic intramural hematoma observed by endoscopic ultrasonography. Endosc Forum Digest Dis 17(2): 162-165.

12. Fischer J, Samson P, Robertson G (2010) Anticoagulant-induced intramural haematoma of the caecum mimicking a colonic tumour. $\mathrm{N} \mathrm{Z}$ Med J 123(1315): 75-78.

13. Kwon CI, Ko KH, Kim HY, Hong SP, Hwang SG, et al. (2009) Bowel obstruction caused by an intramural duodenal hematoma: a case report of endoscopic incision and drainage. J Korean Med Sci 24(1):179-183. 
14. Nakadaira K, Ueki H (2015) A case of transverse colon cancer with intramural hematoma of the ascending colon. J Japan Surg Assoc 76(11): 2754-2759.

15. Nogales Rincón O, Yepes Barreto I, Hernando Alonso A, González Asanza C, Menchén Fernández-Pacheco P (2009) Large intramural colonic hematoma after polypectomy. Endoscopy 41 Suppl 2: E3.
16. Marques I, Lagos AC, Pinto A, Neves BC (2011) Rectal intramural hematoma: A rare complication of endoscopic tattooing. Gastrointest Endosc 73(2): 366-367.

For possible submissions Click below:

Submit Article 